\title{
Testing and validation of the ACCESS system
}

\author{
Kyle JENNETT \\ PNDC - UK \\ kyle.jennett@strath.ac.uk
}

Federico COFFELE

PNDC - UK

federico.coffele@strath.ac.uk
University of Strathclyde - UK

Bruce STEPHEN

bruce.stephen@strath.ac.uk ibrahim.f.abdulhadi@strath.ac.uk

\begin{abstract}
With the increasing penetration of distributed renewable generation being connected, a number of solutions have been developed to avoid network overloads. A number of the already implemented schemes are based on generation curtailment during times of high generation and low demand.
\end{abstract}

The ACCESS (Assisting Communities to Connect to Electric Sustainable Sources) system implements a more sophisticated predictive based solution. In the ACCESS system the generation resource and electrical demand is predicted and the storage heating resource is managed, providing a demand side management instead of a generation curtailment solution.

The ACCESS system was installed, tested and demonstrated in the Power Network Demonstration Centre (PNDC), a research facility that enables realistic testing of novel hardware and network operation schemes in a controlled environment, in 2015. Following the testing at the PNDC, the ACCESS system was deployed in the Isle of Mull, in 2016.

This paper reports on the outcome of the testing of the demand response features of the ACCESS system at the PNDC prior to its deployment on the Isle of Mull. This paper reports on: the different components of the system in detail (i.e. what it is designed to do and why); the test regime undertaken at the PNDC; the results of the testing (i.e. how effectively it achieves its design objectives); and the updates to the ACCESS system based on the testing with regards to the solutions employed and the benefits achieved.

\section{INTRODUCTION}

There are a number of ongoing related areas of demand side management research. In [1], the authors look at predictive modelling of wind power and associated energy storage requirements. In [2], the authors looks at local generation and consumption of energy within a community with marginal energy import/export. Specifically, in [2] the authors looks at monitored data from a community micro-grid system in the UK. In [3] a simulation based case study with operational constraints, wind generation and district heating is presented. The results demonstrate the effectiveness of different sizes of heat store, showing that the most substantial gain is with small storage and only a marginal increase in wind output is achieved with increasing storage size. [4] presents on an energy management system (that aggregates distributed energy resources) on a simulated $11 \mathrm{kV}$ distribution network. [5] gives an overview of various DSM techniques and identifies some of the challenges to DSM deployment (e.g. information security). The ACCESS project is distinct from these papers in that it includes the predictive and energy storage elements in a real world environment.

The first section of this paper describes the objective of the ACCESS system, the second section discusses the testing undertaken at the PNDC, the third section presents the results of the testing and the fourth provides a summary of the ACCESS system updates incorporated as a result of the testing.

\section{ACCESS SYSTEM OBJECTIVE}

The ACCESS system addresses the problem of transmission constraint (thermal power constraint) in islanded and isolated rural networks. These networks are physically isolated from the rest of the UK electrical network and therefore the power distribution infrastructure required to transfer power to and from these communities (e.g. from renewable sources) is constrained. Network reinforcement to remedy this can be an expensive solution in the context of the amount of power being exported. A potentially more cost effective solution is to implement a Demand Side Management (DSM) scheme to use the locally generated power to supply local demand. Challenges associated with implementing an effective DSM scheme include: generator (power output limiting) and load control; instrumentation of the generator and load; management of a communications infrastructure between measurement locations; and implementation of a control algorithm to manage the system.

The ACCESS system allows community generation to be installed in islanded or rural networks without exceeding the transmission constraints on the network. The ACCESS system operates in the role of a 'micro' Distribution System Operator (i.e. managing both generation and demand locally) within the Mull Community Energy Scheme. The ACCESS system addresses the problem of transmission constraint by controlling local demand (in the form of a retrofit controller applied to the heating load) and local generation output in real time as shown in Figure 1. This paper presents a general assessment of the ACCESS generation and demand side management solution within the context of existing power systems. The focus of this 
paper is on the VCharge Dynamo virtual [6] district heating component and the challenges of integrating the different elements of the scheme. After the ACCESS testing programme was concluded and the test results had been fed back to the ACCESS system team (for development) the system was deployed on the Isle of Mull. The ACCESS system is now operational as a trial on Mull [1].

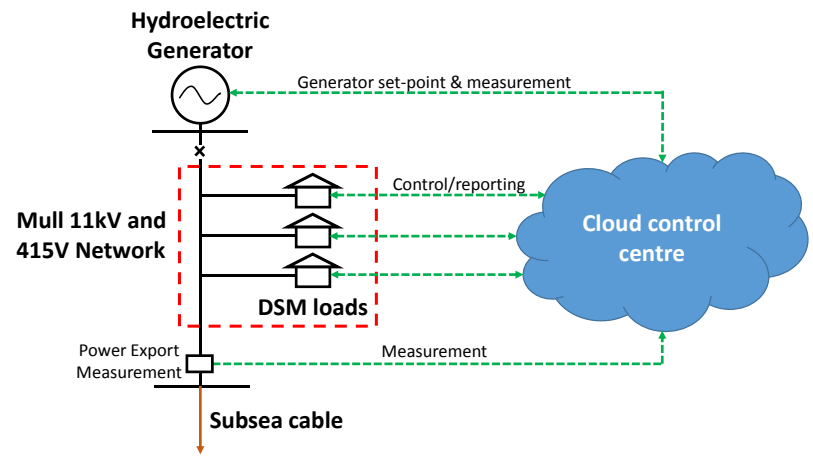

Figure 1 Overview of ACCESS system

\section{PNDC TESTING}

The aim of the PNDC testing was to demonstrate and test the functionalities of the ACCESS demand side management system, following a testing plan agreed with Community Energy Scotland (CES) and Scottish and Southern Energy Power Distribution (SSEPD) Limited. Testing required the preparation of a realistic environment at the PNDC to evaluate the ACCESS system prior to it being deployed in Mull. The test environment included: simulation of the Garmony generator and its Kestral Control System [7]; including establishing the required interfaces for connection to the simulated generator.

The second step in the testing process involved the installation and commissioning of the following hardware:

- The domestic DSM loads: ten Dynamo units (discussed in the following section) controlling ten Dimplex quantum storage heaters, connected to one secondary substation at the PNDC, and a Hub establishing internet communications with the Dynamos through a home router.

- The satellite communications network: two Q-sat satellite systems [10], allowing VPN communication between devices at the simulated measurement point, and those at the simulated generator.

The test program included two components:

1. Power quality testing of the dynamo units

2. End to end testing of the full ACCESS system

\section{Dynamo hardware overview}

The VCharge Dynamo is a permanently installed load control and monitoring device for resistive loads. It is equipped with revenue grade power metering and energy sub-metering, and communicates wirelessly. It is shown in isolation from a heater in Figure 2.

The objective of this test was to determine if the dynamo units switch on and off (when they are controlled do so) and to evaluate the dynamo metering accuracy. Each dynamo unit was evaluated for switching functionality and metering capability. The dynamo units were controlled via 'manual' control to evaluate capability to control the associated loads. In this context, 'manual control' meant controlling the Dynamo directly with a local interface rather than the automatic algorithm control. The metering data sent by the dynamo units to the VCharge Cloud was compared against the power data recorded by the FLUKE 435 Power Quality Analyser [11].

The Dynamo hardware test had three components: a functional test to determine metering accuracy and control; an evaluation of the power quality impact of the Dynamo hardware on the network (specifically harmonic impact and switching); and the response of the Dynamo during communication failure.

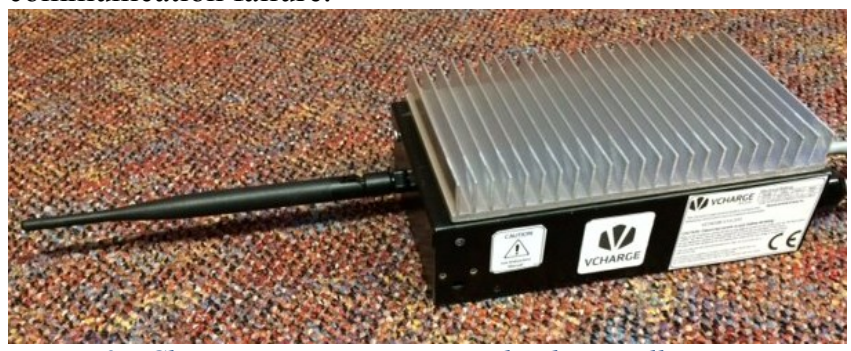

Figure 2 VCharge Dynamo responsive load controller

\section{End to end test overview}

The objective of this test was to integrate the different elements of the scheme and evaluate whether the different components would operate correctly.

This test was achieved using the experimental setup shown in the electrical line diagram in Figure 3. In this test the Garmony Hydro Electric Generator was simulated in a real time digital simulator (RTDS) environment. The generator simulation was interfaced with the VSCon (router to generator interface) over a Modbus RS485 interface. A protocol conversion between the RTDS DNP 3 interface and Modbus RS485 interface was implemented. The VSCon interfaced with the VCharge cloud control centre over a satellite communication interface installed at the PNDC. The VCharge cloud control centre communicates with the Dynamo hardware using a local router installed at the PNDC. 


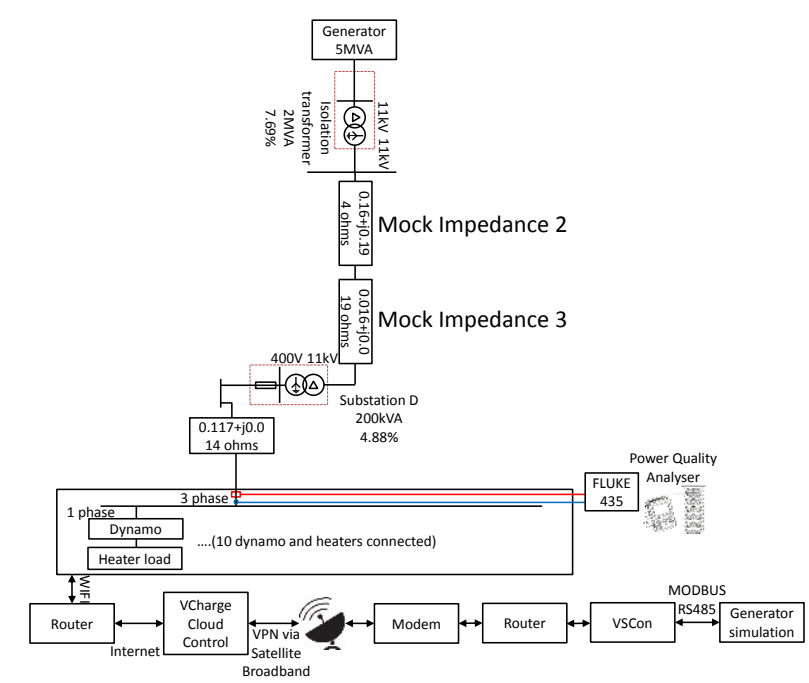

Figure 3 Electrical line diagram of end to end test

\section{TEST RESULTS}

The key results relating to the VCharge Dynamo virtual heating component pertain to the challenges of integration. In order to address this challenge each modular component was tested individually and then integrated in the final 'end to end test'. This paper reports first on the modular component testing of the dynamo unit and then the full system 'end to end test'. The primary challenge of integrating this system was pre-testing and involved the communication links between the ACCESS system components. Specifically this included establishing satellite communication to the dynamo hardware and generator simulation from the VCharge cloud control system. The 'pre-testing' process of de-bugging the communication system is not reported in this paper. During testing the key learnings and challenges associated with operating the ACCESS system related to the erroneous operation of the VCharge cloud control algorithm to the generator behaviour. These findings are reported on this section and were addressed by VCharge in post-testing updates to the system.

\section{Dynamo hardware test results}

The results of the dynamo hardware test are shown in Figure 4. In this test each of the dynamo units were turned on sequentially and the heater load associated with each was logged with the power quality meter. The active power logged by the Fluke 435 matched the metering data recorded by the VCharge Cloud with a maximum error over the test period of less than $\pm 3 \%$ (shown as error bars in Figure 4). This was the expected response from this test.

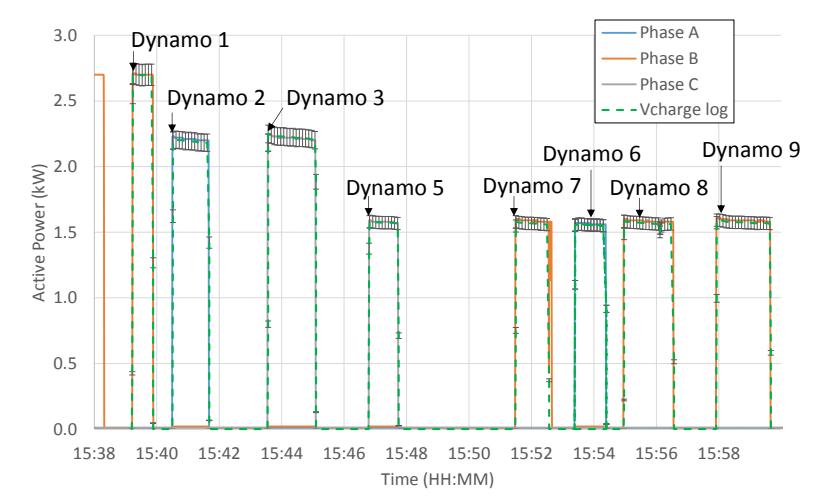

Figure 4 Dynamo functional of dynamos controlled to turn on with manual control signal

\section{End to end test results}

In the end to end test three modes of operation were tested (as reported in the following sections).

\section{Functional test}

Figure 5 shows the results of the end to end functional test: the unconstrained setpoint of the generator (i.e. what the generator is being controlled to do by the VCharge cloud control algorithm and not considering the physical ramp limitations of the generator), the constrained generator output (as applied by the VCharge control algorithm and the physical ramp rate constraints of the generator), and the recorded active power load of the heaters. In this test the unconstrained generator setpoint ramps at a faster rate than the generator can follow so the generator output lags behind the unconstrained output. Ideally the mechanical operation of the generator would operate quickly enough to follow the control signal of the cloud control algorithm.

The unconstrained setpoint of the generator ramps from 0 $\mathrm{kW}$ to $400 \mathrm{~kW}$ over $10 \mathrm{~min}$, sustains an output of $400 \mathrm{~kW}$ for $5 \mathrm{~min}$ and then ramps down to $0 \mathrm{~kW}$ over $15 \mathrm{~min}$. It can be observed that when the generator output reaches 80 $\mathrm{kW}$ the VCharge algorithm controls the heaters to turn on to limit the difference between generation and demand to less than $50 \mathrm{~kW}$. When the generator output reaches 205 $\mathrm{kW}$ there is not enough heating load available to maintain the difference between generation and demand to less than $50 \mathrm{~kW}$ so the VCharge control algorithm constrains the generator output to $205 \mathrm{~kW}$. It should be noted that in this test the available heating load is scaled up in the algorithm so that each heater represents more power than is actually available (155kW of controllable load). The experiment was configured in this way so that the algorithm could be tested for a larger controllable thermal load than existed onsite.

To evaluate a range of load availabilities the maximum brick temperature allowance of one storage heater was reduced. This caused the heaters control system to assume it was fully charged and then to control the heater to stop charging (i.e. to stop consuming active power). The 
VCharge control algorithm identified that the heater was no longer available as a heating load and constrained the generators setpoint to maintain the generation minus heating load limit of $50 \mathrm{~kW}$. This constraint can be observed as a dip in generator setpoint at 15:14 in Figure 5.

There are three unexpected operations in this test (annotated 1-3 in Figure 5). At (1) and (3) the $50 \mathrm{~kW}$ threshold is exceeded. At (2) the generator output is constrained to ramp down and as a result the heating load is also controlled to ramp down. The operation at (2) is unexpected because there is no reason for the generation output to be constrained, the generator should only be constrained if there is insufficient heating load or insufficient generation capacity.

At (1) and (3) (in Figure 5) the $50 \mathrm{~kW}$ threshold limit being exceeded was due to the artificially scaled heater load (i.e. $155 \mathrm{~kW}$ of controllable load). The VCharge cloud control system which is responsible for dispatching the heaters misinterpreted the scaled load value (scaling factor error) resulting in the threshold being exceeded. In an earlier test (i.e. an 'unscaled' load) this was not observed to occur. VCharge have advised that since this test they have updated their system so that it will correctly interpret larger heating loads.

At (2) (in Figure 5) the VCharge cloud control system shows a false negative on the connectivity status with the generator (i.e. a false communication failure alarm). The response of the VCharge system to a loss of communication alarm is to ramp down both generation and load (the response seen in this test). Since this test VCharge have advised they have observed this response with a different Satellite link in Orkney and have now resolved the underlying cause of the false communication failure alarm.

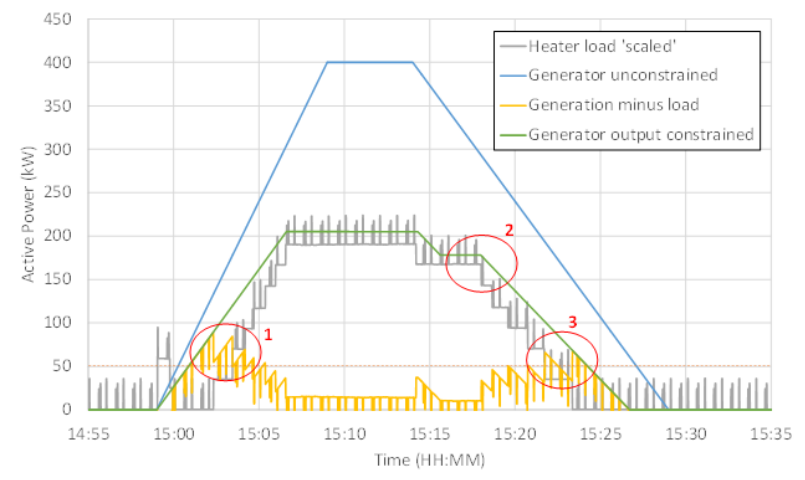

Figure 5 Functional test response of generator output increasing

VCharge (in consultation with the heater manufacturer Dimplex) advised that the regular 'pulses' observed in this test were caused by a fault in the Quantum heater internal code. VCharge advised that Dimplex have now updated the code in their heaters to remove this issue.

\section{Communication failure}

Figure 6 shows the response of the VSCON to a communications failure. In this test The VSCon communication failure was applied at 08:52:10 (by disconnecting the satellite link). At 08:52:20 the VSCon began decreasing the generator setpoint (to reduce the generator output to $50 \mathrm{~kW}$ over $10 \mathrm{mins}$ ).

The VCharge algorithm also detects the loss of communication and switches the heaters to an uncontrolled mode of operation. The change to uncontrolled operation is indicated by the active power load of the heater decreasing to zero. It can be observed from Figure 6 that the heaters do not all transfer to uncontrolled mode simultaneously, the transfer to uncontrolled mode is staggered over a period of approximately $10 \mathrm{~min}$. As the generator output and heating load is reduced (in response to the communication failure) it can be observed that the generation output minus the heater load remains less than $50 \mathrm{~kW}$ at all times. This is the expected response from this test.

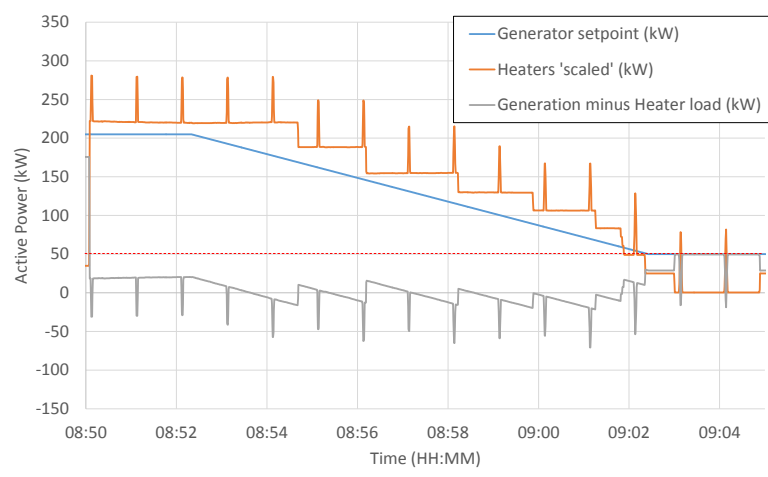

Figure 6 Communications failure test

\section{Generator power control failure}

Figure 7 shows that when the unconstrained generator setpoint reached $58 \mathrm{~kW}$ the VCharge algorithm controls the heating load to ramp up thus reducing the net generator output to less than $50 \mathrm{~kW}$; however, insufficient heating load at this time fails to achieve this so an additional heater load turns on albeit slightly later. The correct operation is that all available heating loads should turn on at the same time to try to reduce the generation minus heating load below $50 \mathrm{~kW}$. 


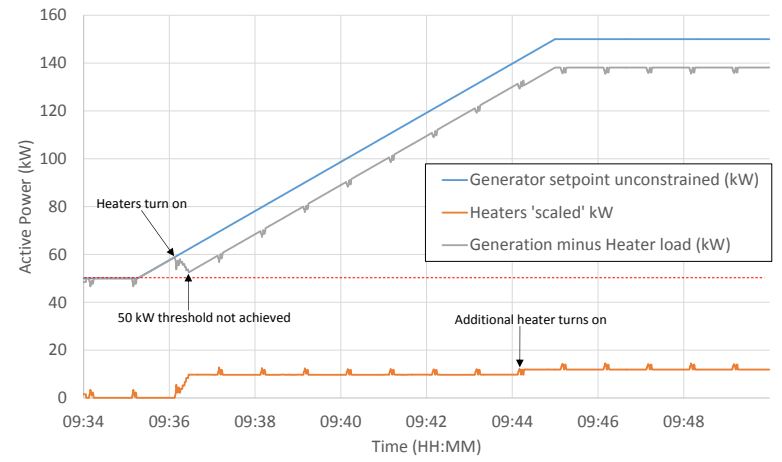

Figure 7 Test recording from generator power control failure test

\section{ACCESS SYSTEM UPDATES POST-TESTING}

The realistic testing conducted with the facilities at PNDC raised operational issues that would have been costly or time consuming to rectify in the field. In particular, limitations of the existing communication protocol between the heaters and the head end system, more resilient communications and issues with the retrofitting of the heating control system. The follow issues were identified during testing and were addressed during the test process or immediately afterward prior to deployment of the system on Mull:

- VCharge updated their control algorithm so that it will correctly interpret larger heating loads.

- The VCharge log shows a false negative on the connectivity status with the generator (i.e. a false communication failure alarm). Since this test VCharge have advised they have observed this response with a different satellite link in Orkney and have now resolved the underlying cause of the false communication failure alarm.

\section{CONCLUSIONS}

This testing validated the response of the ACCESS system from an individual component level up to a fully integrated system. The results from this project revealed a number of areas for improvement that were corrected either during testing or prior to deployment on site in Mull.

The main operational challenge identified through the test program was related to the VCharge control algorithm's response to changes in generation or demand. The novel utilisation of the RTDS (representing the generator in simulation) assisted in identifying this issue at an earlier stage and allowed these problems to be resolved prior to deployment in Mull.

\section{REFERENCES}

[1]

H. V Haghi and S. Lotfifard, "Spatiotemporal modeling of wind generation for optimal energy storage sizing," 2015 IEEE Power \& Energy Society General Meeting. p. 1, 2015.
[2] N. P. A. D, E. H. Owens, S. Roaf, D. W. Corne, M. Dissanayake, B. Stephen, and S. Galloway, "Autarkic Energy Systems : Balancing Supply And Demand with Energy Storage and Controls in Local Energy Micro-grids."

[3] S. Gill, M. J. Dolan, D. Frame, and G. W. Ault, "the Role of Electric Heating and District Heating Networks in the Integration of Wind Energy To Island Networks," Int. J. Distrib. Energy Resour., vol. 7, no. 3, pp. 7138-7138, 2011.

[4] C. Cecati, C. Citro, and P. Siano, "Combined Operations of Renewable Energy Systems and Responsive Demand in a Smart Grid," IEEE Transactions on Sustainable Energy, vol. 2, no. 4. pp. 468-476, 2011.

[5] P. Palensky and D. Dietrich, "Demand Side Management: Demand Response, Intelligent Energy Systems, and Smart Loads," IEEE Transactions on Industrial Informatics, vol. 7, no. 3. pp. 381-388, 2011.

[6] J. Millar, "VCharge." [Online]. Available: http://vcharge-energy.com/. [Accessed: 19-Dec2016].

[7] S. Black, "Kestral Controls Limited." [Online]. Available: http://www.kestralcontrols.co.uk/. [Accessed: 19-Dec-2016].

[8] P. Bas, "The Tollgrade Lighthouse." [Online]. Available: http://www.tollgrade.com/smartgrid/smartgrid-products/overview/. [Accessed: 19-Dec2016].

[9] C. Breaden, "sgs connect." [Online]. Available: www.smartergridsolutions.com/media/32195/co nnect-new-oct.pdf. [Accessed: 19-Dec-2016].

[10] N. Quinn, "QSAT." [Online]. Available: http://www.q-sat.co.uk/. [Accessed: 19-Dec2016].

[11] "FLUKE 435 Series II Power Quality and Energy Analyzer." [Online]. Available: http://www.fluke.com/fluke/uken/powerquality-tools/logging-power-meters/fluke-435series-ii.htm?PID=73939. [Accessed: 19-Dec2016]. 